\title{
Needle Force Sensor, Robust and Sensitive Detection of the Instant of Needle Puncture
}

\author{
Toshikatsu Washio and Kiyoyuki Chinzei \\ Surgical Assist Technology Group, AIST, \\ 1-2-1 Namiki, Tsukuba, Ibaraki, 305-0051, Japan \\ \{washio.t,k.chinzei\}@aist.go.jp
}

\begin{abstract}
A force sensor based method to detect the instant of needle puncture to the layers of tissues is proposed. A set of needle and sheath attached to a pair of coaxial force sensors was used to separately measure the cutting force at the needle tip and the sidewall friction. Change of the force profile was observed and marked at the instant of the puncture, and robustly eliminated artifacts caused by body motion such as the respiratory or cardiac motion. The agreement between the subjective feeling of a skilled surgeon and the sensor was good. In several cases the sensor detected the puncture even when the surgeon could not clearly identify it. It was suggested that the sensor is potentially more sensitive than skilled professionals.
\end{abstract}

\section{Introduction}

Needle insertion is one of basic medical skills, and also one of the recent technology challenges for robotics. Though image guidance may be applied when inserting a needle to a critical organ, the essential information for good insertion is the subjective feeling from the needle.

Many attempts of robotic needle insertion [1-4] as well as the simulation of puncture [5-8] have been reported in literature. Generally, it is desirable that a robotic needle drive be equipped with at least one monitoring method for safety. The majority of approaches perform the insertion with the aid of an imaging device $[1,2]$. Some of them monitor the insertion with a force sensor instead of imaging $[3,4]$. Force sensing has no spatial information, though it has advantages over imaging in the view of its ability to sense the dynamic and local properties of tissue [9] and its great cost effectiveness.

The goal of this study is to establish a sensing method of needle insertion that is applicable to robotic needle insertion. In reality, it is not easy to sense the tissue properties using a force sensor, due to the disturbance from body motion, including respiratory and cardiac motion. These can be often dominant in the signal profile of the force sensor.

For this problem, the authors developed coaxial force sensors attached to the coaxial needles. Using this system, we found that the instant of needle puncture was stably detected without being affected by the artifacts. We also examined that the subjective feeling of needle puncture by a skilled medical professional agreed with 
the sensor's signal pattern. It was also suggested that the sensor could detect the instant of the puncture better than the skillful professional.

This paper is organized as follows: Section 2 outlines the structure and the principle of the sensor system with the underlying assumptions. Section 3 and 4 describe the experiments on the stability against the artifacts, the agreement with the subjective feeling, followed by a discussion and conclusion.

\section{Sensor and Needle Structure for Stable Sensing}

\subsection{What Do Skilled Professionals Feel?}

To date, the skill of needle insertion indeed falls in the realm of art. Though it is not trivially clear that skilled professionals sense needle insertion as force, we assume that we can obtain relevant information about needle insertion by force measurement.

The next question is what information they obtain from the force. Skilled professionals can insert a needle from the skin to the epidural space without image guidance. When they do it, they 'monitor' the puncture of layers where they expect to pass through: the skin, the muscle, the ligament, etc. The passage of layers may be detected by two ways: difference of the mechanical properties such as stiffness, or characteristic pattern at the instant of puncture of the membrane between the layers. We consider the latter in this paper and we assume that the break of the membrane is sensed as the force at the needle tip.

Unfortunately, the information through the needle does not carry the force at the needle tip alone. The force is composed of the force at the needle tip and the friction to the needle sidewall. The latter works as noise that occludes the former. As the needle is inserted deeper, it is expected that the friction increases and sensing the needle insertion becomes difficult.

\subsection{Structure of the Sensor and the Needle}

Based on an early study with our collaborators [10], the authors developed a set of coaxial load cells that can attach a coaxial needle where a sheath covers an internal needle so that only the blade part of the latter interfaces to the tissue (Fig. 1). Both the sheath and the blade are individually attached to the load cells. Since the sheath and the blade are independently attached to the load cells, the sensors basically represent the sidewall friction and the needle tip force respectively.

\subsection{Detection of Puncture (Algorithm)}

When a needle tip touches a membrane between two different layers of tissues, it reaches the puncture after certain extension. When the membrane breaks, its tension is released and the membrane and the tissues recover the original shape. At the same time, the needle proceeds into the tissue as the consequence of the recovery. Figure 2 
illustrates the behavior when a needle breaks the skin, which is a special case of the puncture between two layers.

At the instant of the puncture, both sensors are expected to behave as the following. While the tension of the membrane is released, the needle tip cuts into the tissue. In case of the puncture of soft tissues, the strength of the membrane is usually superior to that of tissue, therefore the total force is expected to decrease. For the sheath, the sidewall friction will steeply increase in accordance to the needle penetrating the tissue.

In summary, at the instant of the puncture, we expect to observe that the tip force decreases and the friction increases. This does not occur due to body motion, since the expected effect of such motion is to either increase or decrease both forces at once.

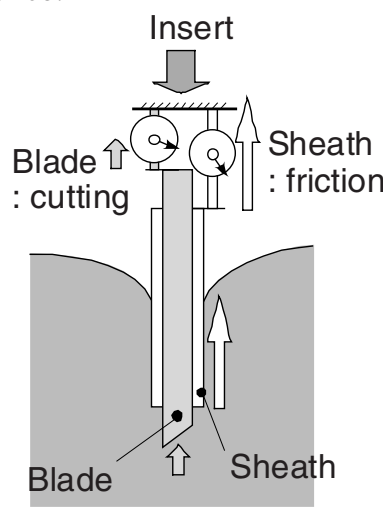

Fig. 1. Structure of the needle force sensor.

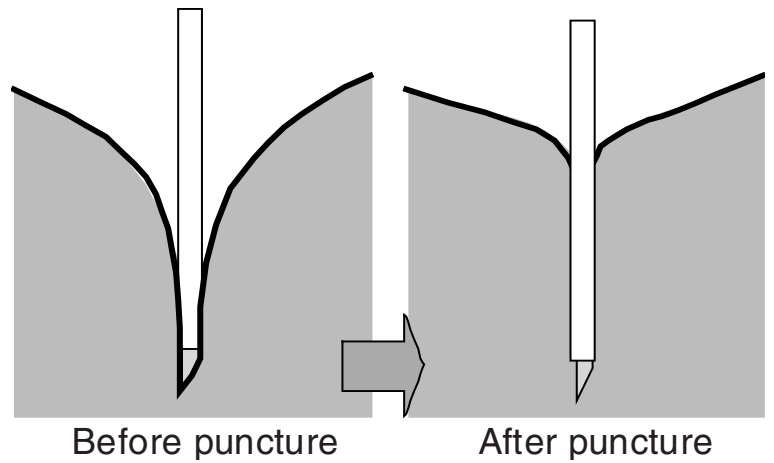

Fig. 2. The instant of puncture. As the membrane breaks, the membrane and the tissue recover the original shape. Consequently the needle proceeds into the tissue.

\section{Validation}

To validate the sensor, two experiments were performed; 1) Specificity of the detection of puncture under the existence of the respiratory and cardiac motion, 2) Sensitivity of the sensor and agreement with the detection to the subjective feeling.

Figure 3 REFshows our prototype of the coaxial load cell. For experimental purposes, the load cell attached to the sheath is a 6-axes force sensor, though in this paper the axial force component alone was used and discussed. Figure 4 shows a coaxial needle attached to the sensor, $130 \mathrm{~mm}$ length and $2 \mathrm{~mm}$ diameter. It is known that the shape of the needle tip affects the needle insertion. We examined several different needle tips. We examined by preliminary experiments that the shapes of needle tip did not affect the findings in this paper.

\subsection{Detection and Its Stability Against Body Motion}

The sensor was fixed to a linear motion table. The needle was inserted into a porcine liver under anesthesia. The liver was exposed in advance to the experiment, and was 
periodically moved by the respiratory motion. The needle was inserted by a constant speed of $5 \mathrm{~mm} / \mathrm{sec}$. Since the point where the needle touches was not always visible, a white plastic tube of $2.5 \mathrm{~mm}$ inner diameter, $35 \mathrm{~mm}$ length and $720 \mathrm{mg}$ weight was placed on the surface of the organ so that the needle went through it. Motion of the tube was observed by video to determine the instant of puncture. It was determined as the puncture when the tube started moving backward according to the recovery of the tissue to its original shape.

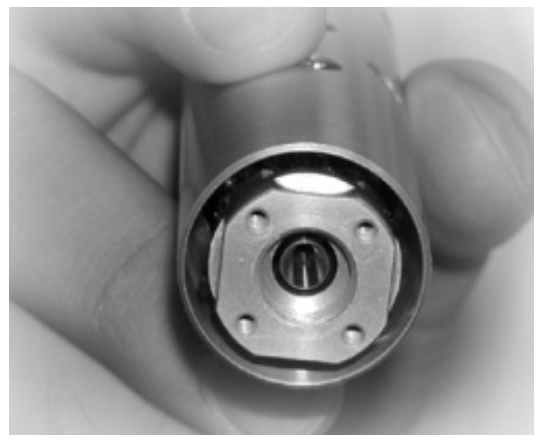

Fig. 3. A prototype of the coaxial sensor, 6-axes (external) + 1-axis (internal).

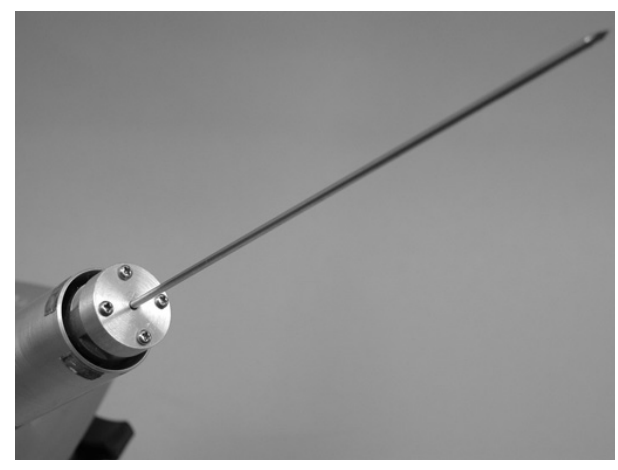

Fig. 4. The coaxial needle attached to the sensor.

The insertions were performed over 45 cases. The characteristic pattern described in section 2.3 was sought by visual inspection and defined as the detection of the puncture by the sensor. The time of the puncture detected by the sensor relative to that of the video observation was examined.

\subsection{Agreement with Subjective Feeling}

Correlation between the sensor signal and subjective feeling of a skilled surgeon was examined. A skilled neurosurgeon manually inserted the needle by holding the sensor to porcine spleen. We determined the instant of puncture by the video-based detection, i.e., observing the motion of the white tube as stated in section 3.1. When the surgeon felt the puncture occurred, he verbally described it.

The insertions to the spleen were conducted over 59 cases. The instant of the puncture detected by the sensor and declared by the surgeon, both relative to the video-based detection, was examined.

\section{Results}

\subsection{Detection and Its Stability Against Body Motion}

A typical profile of the measured forces is shown in Fig..5. The left figure is the measured profile. The profile pattern of the decrease of the tip force and the increase of the side friction appeared at $\mathrm{t}=8$, which corresponds to the video-based detection. There was another similar pattern at $\mathrm{t}=8.8$. We discuss it in a later section. 
The right figure is the profile of numerical sum of these forces, which simulates observation using a single sensor. The change at $t=8$ was minor in contrast to the effect of the artifact by the body motion.

Figure 6 shows an experimental set-up of constant speed insertion. Disparities of the instant of the sensor detection from the video-based detection were in Fig. 7. The sensor detection occurred up to 1 second earlier than the video-based detection.

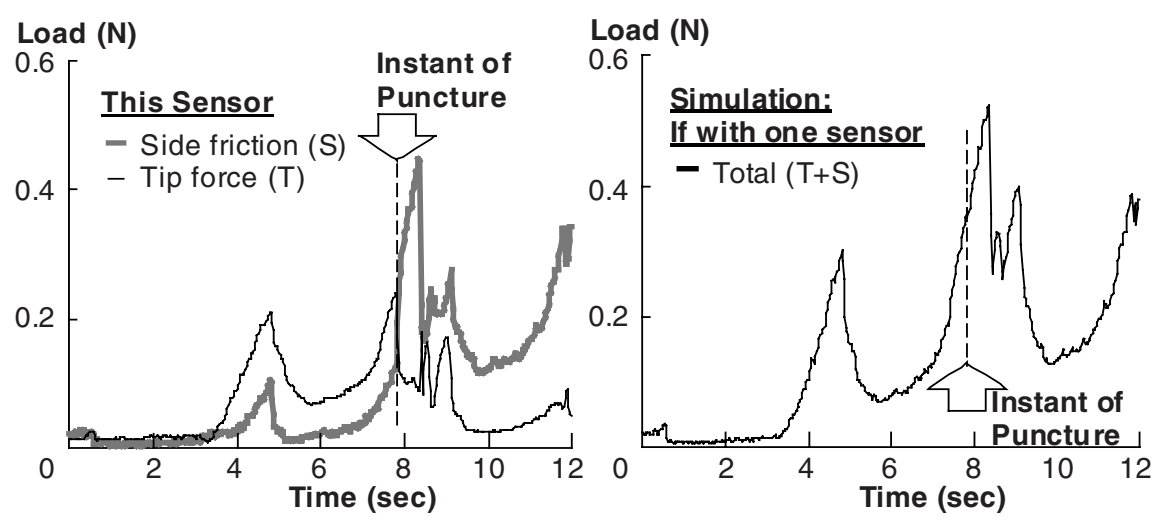

Fig. 5. The profile of the forces by the constant speed insertion. The left profile is the obtained result and the right was the sum of two curves in the left to simulate a single sensor. In the left profile, a periodic change by the respiratory motion mounted in the both curves. At the instant of the puncture $(\mathrm{t}=8)$, the tip force reduced and the side friction increased. In contrast, a single sensor would not be able to detect it under the existence of the periodic change.

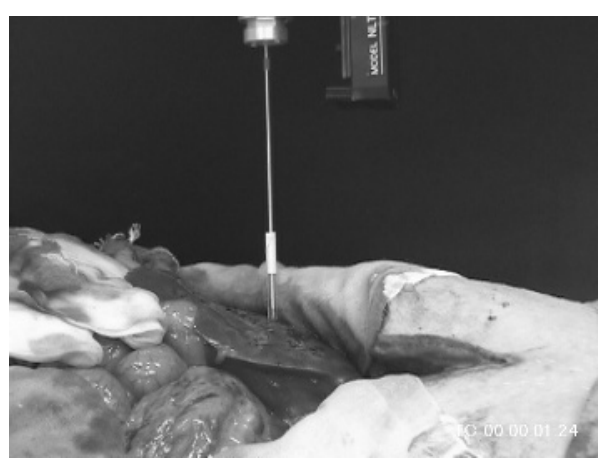

Fig. 6. Experimental set-up of constant speed insertion.

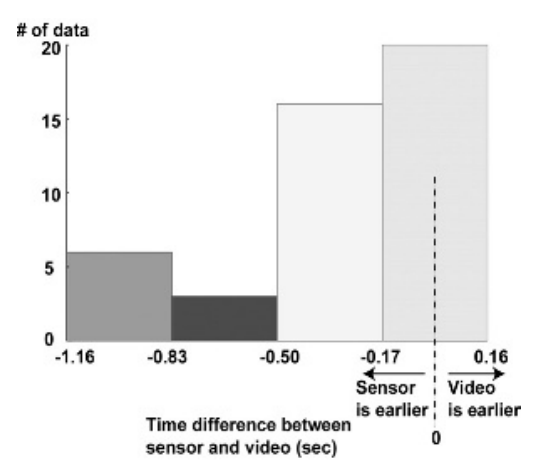

Fig. 7. The histogram of the time difference of the puncture relative to the video detection. The sensor detection started up to 1 second earlier than the surface motion. 


\subsection{Agreement with Subjective Feeling}

Figure 8 shows an experimental set-up of manual insertion. A distribution chart was obtained for 20 of 59 insertions (Fig. 9). The correlation coefficient was 0.88 . In the remaining cases, puncture was not clearly felt by the surgeon or the white tube was occluded therefore unable to determine the video detection. These were removed from the distribution chart.

Figure 10 shows two typical profiles. The left profile is a case that the surgeon described clearly felt the puncture, while the right is a case that he could not feel the puncture. Both profiles exhibit the pattern of puncture occurs 0.28 and 0.17 second earlier than the video-based detection.

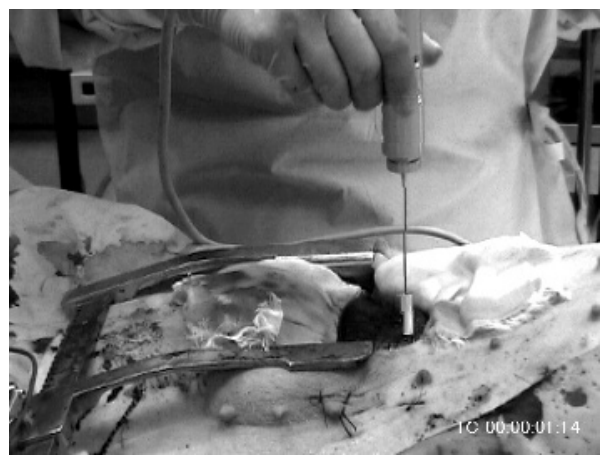

Fig. 8. Experimental set-up of manual insertion.

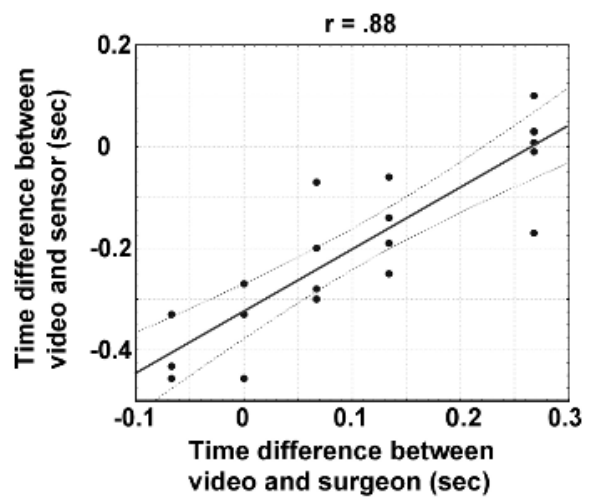

Fig. 9. The distribution chart of the time difference of puncture detected by the sensor and felt by the surgeon.
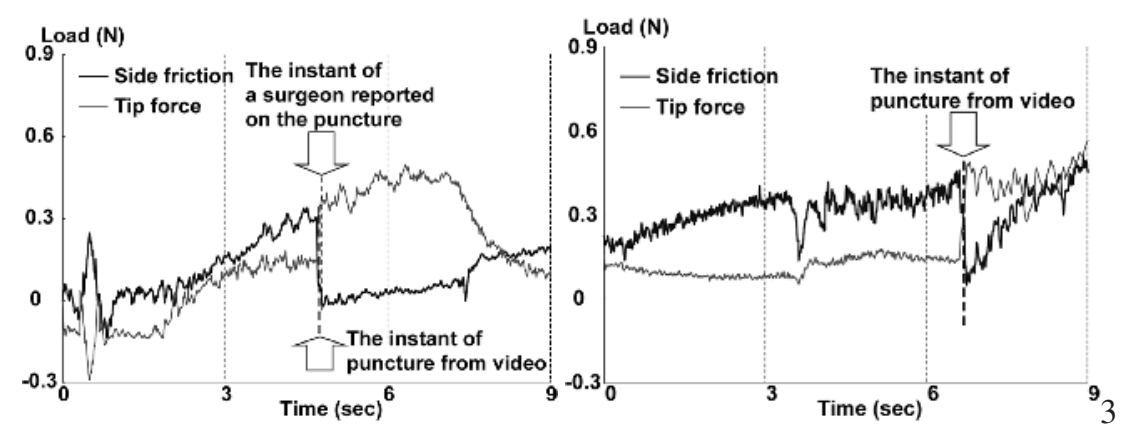

Fig. 10. The profile of the forces by the manual insertion to the spleen. The left profile is a 'clearly felt' case and the right is a 'unclear case he could not find when the puncture occurred'. However, the sensor signal has the pattern similar to the puncture. 


\section{Discussions}

The observed force profile indicated that the artifact from the body motion could easily surpass the change caused by the needle puncture (Fig. 5). The overall performance was excellent in suppressing the effect of the artifacts. There was another similar pattern at $\mathrm{t}=8.8$ in Fig.. 5. Its cause was not identified, though it is estimated that we detected another puncture of the internal structure of the liver.

The agreement between the detection by the sensor and visually determined instant of puncture (Fig. 7) showed that 1) almost all of the sensor detection precedes the video detection, 2) the time difference could reach 1 second. The former fact justified the assumption in Section 2.3. The authors consider that the delay can largely depend on the phase of body motion and the hysteresis of interaction between the needle and tissue. It is suggested that the video detection can be used as the baseline, however it has a window of 1 second in the detection time.

The agreement between the sensor output and the subjective feeling was in general good (Fig. 9). The response of human subject had a constant delay of 0.23 second from the sensor detection. The authors consider it is a typical delay for a human subject responding to a sensory input.

The profile in Fig. 10 shows another interesting event, that the case of the right figure was described as 'unclear when the puncture occurred'. However, there is a pattern of the puncture in the force profile that was 0.28 second preceding to the video based detection of the puncture. It is suggested that the sensor is more sensitive than a skilled surgeon. Regarding the sensitivity, the authors plan different experiments to evaluate the limitations of the sensor.

Since the experiments were performed on the surface of tissues, further study is required to conclude that this method can detect the puncture of layers of different tissues. We also plan to validate the agreement between the sensor and the subjective feeling with more subjects in different situations.

The interpretation of the sensor outputs, one for the cutting force and another for the friction, should be understood as an approximation. Strictly, both the sheath and the needle are in contact with each other; therefore, the separation of these forces is not perfect. When the needle is deflected, the bending force can be applied to both sheath and needle as a counter-force, which can theoretically appear as the pattern of the insertion.

Our detection algorithm needs further improvement. In this study we identified the puncture pattern by observing the force profile. We are currently developing computational method to detect it.

The method described in this paper has potentials in application for safety as well as the logging of manual needle insertion, safety monitoring in combination with robotic tools. For basic science, it will contribute to study the secret of how skilled professionals feel and control the needle insertion. 


\section{Conclusion}

A sensor for detecting the instant of the needle puncture was proposed. A pair of coaxial force sensors attached to a set of needle and sheath was evaluated in terms of its robustness against the artifacts by body motions and the agreement with the subjective feeling of a skilled medical professional.

At the instant of the puncture on the surface of the tissue, the output from the sheath increased while that of the needle decreased. This pattern was observed regardless to the manual or robotic insertion. The sensor detection had a good agreement with the video-based detection. When the surgeon could clearly felt the puncture, the agreement between the sensor and subjective detections were also good. In some cases, while the surgeon could not clearly felt the puncture, the sensor detected the pattern and agreed with the video-based detection. It was suggested that the sensor has the potential to sensitively detect the needle puncture better than skilled professionals.

\section{Remarks}

These experiments had been supervised and approved by the internal review board of AIST for animal experiments. Trained veterinarians supported the animal experiments and the animals were treated in the humane manner.

\section{References}

1. fichtinger, G., Krieger, A., Susil, R. C., Tanacs, A., Whitcomb, L. L., Atalar, E.: Transrectal Prostate Biopsy Inside Closed MRI Scanner with Remote Actuation, under Real-Time Image Guidance. Proc. MICCAI 2002. (2002) 91-8

2. Hong, J., Dohi, T., Hasizume, M., Konishi, K., Hata, N.: A Motion Adaptable Needle Placement Instrument Based on Tumor Specific Ultrasonic Image Segmentation. Proc. MICCAI 2002. (2002) 122-9

3. Zivanovic, A., Davies, B.L.: A Robotic System for Blood Sampling. IEEE Trans. on Information Technology in Biomedicine. 4(1). (2000) 8-14

4. DiMaio, S.P., Salcudean, S.E.: Needle Steering and Model-Based Trajectory Planning. Proc. MICCAI 2003. (2003) 33-40

5. Brett, P.N., Parker, T.J., Harrison, A.J., Thomas T.A., Carr, A.: Simulation of resistance forces acting on surgical needles. Proc Instn Mech Engrs. 211(H). (1997) 335-47

6. Kwon, D., Kyung, K., Kwon, S.M., Ra, J.B., Park, H.W., Kang, H.S., Zeng, J., Clealy, K.R.: Realistic Force Reflection in a Spine Biopsy Simulator. Proc. IEEE ICRA 2001. (2001) 21-6

7. Popa, D.O., Singh, S.K.: Creating Realistic Force Sensations in a Virtual Environment: Experimental System, Fundamental Issues and Results. Proc. IEEE ICRA 1998. (1998) 59-64

8. Simone, C., Okamura, A.M.: Haptic Modeling of needle Insertion for Robot-Assisted Percutaneous Therapy. Proc. IEEE ICRA 2002. (2002) 2085-91

9. Brett, P.N., Harrison, A.J., Thomas, T.A.: Schemes for the Identification of Tissue Types and Boundaries at the Tool Point for Surgical Needles. IEEE Trans. on Information Technology in Biomedicine, 4(1). (2000) 30-6

10. Kataoka, H., Washio, T., Chinzei, K., Mizuhara, K., Simone, C., Okamura, A.M.: Measurement of the Tip and Friction Force Acting on a Needle during Penetration. Proc. MICCAI 2002. (2002) 216-23 